\title{
Postkeynesianismus. Ein heterodoxer Ansatz auf der Suche nach einer Fundierung: Kommentar zum Beitrag von Arne Heise
}

\author{
Johannes Becker
}

Online publiziert: 28. Februar 2019

(C) List-Gesellschaft e.V. 2019

Ich bin, das sei hier gleich zu Beginn festgestellt, kein Makroökonom. Die Bitte der Herausgeber, den vorangehenden Text zu kommentieren, verstehe ich daher als Aufforderung, die Perspektive des ausgebildeten Laien einzunehmen, des interessierten Zuhörers einer Debatte, die ja nicht nur die Insider der Makroökonomik, sondern auch Vertreter benachbarter Disziplinen, Wissenschaften, Studierende, Journalisten und - bedauerlicherweise - die Kommentatoren in Online-Outlets bewegt. Vielleicht ist diese Laienperspektive sogar eine hilfreiche, denn wenn der von Arne Heise geforderte Wandel der Makroökonomik eintreten soll, so wird dies wohl nur gelingen, wenn eine relativ breite Schicht von Ökonominnen und Ökonomen von seiner Notwendigkeit überzeugt ist.

Dass dies bislang nicht der Fall ist, hat vermutlich eine ganze Reihe von Gründen, darunter aber nicht zuletzt, dass die Kritik an der VWL im Allgemeinen und der „herrschenden“" Makroökonomik im Speziellen selbst nicht unangreifbar ist. ${ }^{1}$ In gewisser Hinsicht hat dies auch strukturelle Gründe; es ist nahezu unmöglich, sinnvoll über die Gesamtheit einer Wissenschaftsdisziplin zu sprechen. Weil die Wirklichkeit komplex ist und Debattenbeiträge in Länge und Tiefe begrenzt sind, besteht die Notwendigkeit zur Vereinfachung - was bewusst oder unbewusst als Rechtfertigung genutzt wird, alles über einen Kamm zu scheren und ein Zerrbild der Realität zu zeichnen.

\footnotetext{
1 Dazu mit leicht anderem Fokus: Becker (2017a, 2017b).
}

This commentary article refers to the article available online at https://doi.org/10.1007/s41025-01900114-x. An author's reply to this commentary article is available online at https://doi.org/10.1007/ s41025-019-00133-8.

\section{J. Becker $(\bowtie)$}

Westfälische Wilhelms-Universität Münster, Münster, Deutschland

E-Mail: johannes.becker@wiwi.uni-muenster.de 
So ist alles, was über die (Makro-)Ökonomik allgemein geschrieben wird, gleichzeitig wahr und falsch, weil alle Beobachter unterschiedliche Blickwinkel haben, individuelle Literaturausschnitte kennen (wer hat schon alles gelesen?) und es beliebig viel anekdotische Evidenz gibt, die alles mögliche belegen kann. Tatsächlich ist dies eine beliebte Strategie im Schlagabtausch zwischen dem Makro-Establishment und seinen Kritikern: eine möglichst breite Gruppe zu definieren (,die etablierten Makroökonomen“) und sie dann über einige wenige Texte oder öffentliche Äußerungen ausgewählter Vertreter zu charakterisieren. Das muss unproduktiv bleiben - und in der Tat weist die Debatte kaum Fortschritte auf. Im Gegenteil, nach einer langen Phase achselzuckender Ratlosigkeit seitens der etablierten Ökonomik, die sich von den pauschalen Anwürfen ihrer Kritiker zumeist falsch repräsentiert ${ }^{2}$ fühlte, keilt die Branche nun zurück: ,People who don't like dynamic stochastic general equilibrium (DSGE) models are dilettantes. By this we mean they aren't serious about policy analysis." (Lawrence et al. 2018)

Fruchtbar wird es immer dann, wenn sich die Kritik auf konkrete Arbeiten, Theorien, Modelle bezieht. In diesem Sinne werde ich mich im Folgenden auch nicht mit dem Postkeynesianismus allgemein auseinandersetzen - dazu fehlt es mir schon, wie oben erwähnt, an Expertise -, sondern speziell mit Arne Heises Text.

Heise nähert sich dem Postkeynesianismus aus der Perspektive des Dogmenhistorikers, der in beeindruckender Belesen- und Gelehrtheit zunächst in der Absicht eines produktiven Scheiterns versucht, das Forschungsfeld des Postkeynesianismus zu gliedern und zu definieren. Das Sortieren, Ordnen und Kategorisieren erfordert eine gewisse Neutralität, der sich unterwerfend Heise auch weitestgehend eine eigene Positionierung innerhalb der postkeynesianischen Theorienwelt vermeidet. Um ehrlich zu sein, macht mir dies meine Aufgabe nicht leichter. Mit der Absicht, ein Mindestmaß an Reibung zu erzeugen, werde ich also im Folgenden einige von Heises Aussagen so interpretieren, als seien sie Ausdruck seiner postkeynesianischen Haltung (und nicht die beispielhafte Erwähnung einer postkeynesianischen Haltung).

Der Postkeynesianismus, so Heise, fasse im Wesentlichen ,verschiedene Theorielinien zusammen (...), die die gleichermaßen das Ziel verfolgen, die in der Wissenschaft seit Jahrzehnten dominanten (dynamisch-stochastischen) Allgemeinen Gleichgewichtsmodelle (DSGM) kritisch zu hinterfragen und als Mainstream abzulösen." (Heise 2019) Dieser Punkt, der später noch einmal wiederholt wird, ist erklärungsbedürftig, denn - zumindest nach meinem Verständnis - handelt es sich bei DSGEModellen weniger um eine eigene Theorie, als um eine Methode bzw. Sprache, um Theorie zu beschreiben. Das bei Heise in Abschn. 4 dargestellte Modell ist auch eine Art allgemeines Gleichgewichtsmodell; und wenn man dem Postkeynesianismus nicht unterstellen möchte, dass er sich an der Stochastik oder an der Dynamik stört (was ihn wohl unmittelbar uninteressant werden lassen würde), dann bleiben zwei Möglichkeiten, den Widerstand gegen DSGE-Modelle zu erklären: ein Missverständnis des Begriffs Gleichgewicht und die Mikrofundierung.

Der Begriff Gleichgewicht ist ein steter Quell von Missverständnissen, das weiß jeder, der ökonomische Theorie unterrichtet. Dass ein Gleichgewicht nicht gut sein muss, lernen die Studierenden spätestens am Beispiel des Gefangenendilemmas,

\footnotetext{
2 Eine lesenswerte Replik auf die Kritik liefert Reis (2018).
} 
und doch trifft man auch bei höheren Semestern immer wieder auf die Vorstellung, mit dem Gleichgewicht verbinde sich etwas Gutes oder Wünschenswertes. Daher verwundert es nicht, dass dieses Missverständnis auch allgegenwärtig in der politischen Debatte ist. So werden die deutschen Exportüberschüsse als bedenkliches Ungleichgewicht gebrandmarkt, obwohl alle theoretischen Erklärungsversuche selbstverständlich auf Modelle verweisen, in der die Exportüberschüsse als Gleichgewicht (bzw. Teil eines dynamischen Gleichgewichts) beschrieben werden. Gleichgewicht im Sinne der ökonomischen Theorie ist also ganz allgemein die Teilmenge all jener Allokationen, die ein Modell aus der unendlich großen Menge aller denkbaren Allokationen erklären kann. Somit bedeutet die Betrachtung eines allgemeinen Gleichgewichts eben nicht, dass damit unterstellt wird, dass die Wirtschaft in ein „gutes“ Gleichgewicht (mit Vollbeschäftigung) zurückkehrt. Im Gegenteil, Gleichgewichte können jede Form wirtschaftlichen Unbills abbilden.

Ähnlich überbewertet ist die Kritik an der Mikrofundierung. Deren Anliegen ist, dass die Bewegungen der aggregierten Größen mit plausiblen Annahmen über individuelles Haushalts- bzw. Unternehmensverhalten vereinbar ist. Letzteres bedeutet nicht, dass man den in der Literatur häufig verwendeten hyperrationalen, unendlich in die Zukunft schauenden Optimierer akzeptieren muss. Aktuelle Forschungsarbeiten wenden sich auch eingeschränkt rationalen Akteuren, Daumenregel-Verhalten etc. zu. Bei dieser grundsätzlichen Flexibilität mikrofundierter Makromodelle ist es aber nur eine Frage der Fantasie, ob sich die Aggregatbewegungen als aggregiertes individuelles Haushaltsverhalten darstellen lassen. So ist die klassische keynesianische Konsumfunktion ähnlich einer Daumenregel, nach der ein fester Bestandteil des Einkommens konsumiert wird. Angus Deaton und viele andere haben gezeigt, dass dies empirisch nicht zutrifft - doch theoretisch mikrofundieren lässt sich solches Verhalten schon. Vielleicht ist es auch hilfreich, sich nochmals vor Augen zu führen, dass das Projekt der Mikrofundierung keiner übertriebenen Vorliebe für eine gewisse Modellästhetik geschuldet ist. Sein Ausgangspunkt war vielmehr die Erfahrung, dass die in normalen Zeiten stabilen Aggregatszusammenhänge in den 70er Jahren plötzlich in sich zusammenbrachen. Erklärtes Ziel der damaligen Makroforschung war eine Theorie, die beide Phänomene - normale Zeiten und Zusammenbruch erklären konnte. Selbst wenn man der Ansicht ist, dass dieses Projekt bislang kein voller Erfolg war (eine Meinung, die selbst bei den Silberrücken des Fachs ja nicht auf großen Widerstand stößt), ist die Rückkehr zu der Mechanistik alter keynesianischer Modelle keine befriedigende Reaktion.

Der US-Ökonom Roger Farmer, der sich selbst als Vertreter postkeynesianischer Forschung versteht schlägt daher schon aus pragmatischen Gründen vor, DSGEModelle nicht abzulehnen, sondern sie als Vehikel für die Verbreitung postkeynesianischer Ideen zu nutzen. „I urge you to think hard about joining me in establishing post-Keynesian DSGE theory as the future of macroeconomics." (Farmer 2017, S. 184) Farmer erkennt damit an, dass es sich bei DSGE-Modellen nur um eine Form der Theorieübermittlung handelt: „General equilibrium theory, broadly interpreted, like mathematics, is a language.“ (S. 184) Postkeynesianische Ideen in diese Sprache zu übersetzen macht sie einem breiteren Publikum zugänglich, setzt sie aber auch dem grellen Licht der akademischen Kritik aus. Krugman beschreibt am Beispiel des Werks von Albert Hirschman, wie dieses nahezu vergessen wurde, weil 
Hirschman sich weigerte, dem Formulisierungstrend in der Ökonomik zu folgen. Doch dann wurde sein Werk wiederentdeckt: „And yet in the end it turned out that mainstream economics eventually did find a place for high development theory. (...), mainstream economics discovered that as its modeling techniques became more sophisticated some neglected insights could be brought back in." (Krugman 1994) Ähnlich erging es dem Werk von Josef Schumpeter, das eine Renaissance feierte, als Ökonomen wie Philippe Aghion und Peter Howitt die Schumpetersche Theorie in ein Modell ,übersetzten“. Warum sollte ähnliches nicht auch beim Postkeynesianismus gelingen?

Ein Charakteristikum des Postkeynesianismus, das der herrschenden Lehre in einem wichtigen Punkt widerspricht und in die DSGE-Sprache übersetzt werden könnte, ist die besondere Rolle von Erwartungen. ,Erwartungen spielen eine überragende Rolle, wobei diese nicht ,rational' im Sinne der ,Rationalen Erwartungshypothese“ geformt werden können“ (Heise 2019). Wenn man, wie Farmer vorschlägt, Erwartungen als Fundamentaldatum akzeptiert, können alle möglichen Gleichgewichte entstehen, darunter auch solche, die dauerhaft Arbeitslosigkeit und ungenutzte Ressourcen implizieren. Demnach wäre ein ,Vollbeschäftigungsgleichgewicht nicht wahrscheinlicher (...) als die Vielzahl von Gleichgewichten auf den Güter- und Geldmärkten, die mit unfreiwilliger Arbeitslosigkeit einhergehen“ (Heise 2019) Anders als in neukeynesianischen Modellen zumeist angenommen, hängt dies aber nicht an Preisrigiditäten oder anderen Marktunvollkommenheiten, sondern schlicht an selbsterfüllenden Erwartungen über das gleichgewichtige Produktionsniveau der Ökonomie.

Solche Hinzugaben der „Expectation Fairy“, um einen Krugman’schen Begriff zu borgen, sind aus Sicht der Theoriebildung ein zweischneidiges Schwert. Zum einen können sie die Prognosefähigkeit verbessern, zum anderen aber müssen die Fundamentalerwartungen auf nicht-tautologische Art beobachtet werden können, um sie tatsächlich zur Prognose verwenden zu können. ${ }^{3}$ Wer sagt aber, dass das nicht gelingen könnte? ${ }^{4}$

Anwendungsmöglichkeiten einer solchen Theorie gäbe es ja genug. Warum zum Beispiel ist die Investitionstätigkeit in Deutschland so gering, obwohl die Wirtschaft nahe der Vollauslastung ist und die Kapitalkosten auf niedrigem Niveau? Befinden wir uns in einem ,schlechten“ Erwartungsgleichgewicht, aus dem die Wirtschaft von selbst nicht herausfindet?

Es mangelt nicht an aktuellen „Puzzles“, an denen sich postkeynesianische Theorie erproben kann. Doch genau diese Herausforderungen muss sie suchen und bestehen. Mich als Außenstehenden interessiert weniger die lange stolze Tradition des Postkeynesianismus im 20. Jahrhundert als die Frage, ob es eine valide, ernst zu nehmende Alternative zur (wie immer auch definierten) Mainstream-Makro gibt. Letztlich entscheidet über die intellektuelle Attraktivität einer Theorie wie dem Postkeynesianismus, ob sie in der Lage ist, empirische Phänomene besser zu erklären

\footnotetext{
3 ,Die Wirtschaft liegt am Boden, weil die Erwartungen schlecht sind; und die Erwartungen sind schlecht, weil die Wirtschaft am Boden liegt" ist keine sinnvolle Theorie.

4 Roger Farmer scheint einen Ansatz zu verfolgen, bei dem die Erwartungen aus Bewegungen von Aktienkursen abgeleitet werden, die dann wiederum Auswirkungen auf die Pläne der Haushalte haben.
} 
als bestehende Ansätze. Heise stellt diese Behauptung allerdings, wenn überhaupt, nur indirekt auf. Stattdessen verweist er, etwas verklausuliert, auf die Überlegenheit der postkeynesianischen Annahmen:

Inwiefern ,sich der Postkeynesianismus in methodologischer Hinsicht von seinem neoklassischen Widerpart unterscheidet: Einerseits kann dies in der spezifischen Variante des positivistischen Fallibilismus bestehen, die als kritischer Realismus besondere Anforderungen an die Validität der Annahmesetzungen stellt (...), andererseits wird explizit auf die Grenzen des Optimierungskonzeptes des methodologischen Individualismus angesichts eines Informationsdefizits verwiesen und mit der besonderen Betrachtung von konventionellen Handlungsroutinen, eingeschränkter Rationalität und alternativen Entscheidungsverfahren (...) ein holistischer Ansatz verfolgt (...). “ (Heise 2019)

Vermeintlich realistischere Annahmen allein lösen das Problem jedoch nicht; es wird darauf ankommen, ob postkeynesianische Modelle einen besseren Datenfit erzielen als andere Modelle. Vor allem aber ignoriert dies die aktuelle Forschungsliteratur im Bereich „Behavioral Macroeconomics“, in der sich mittlerweile sogar die etablierten Größen der herrschenden Lehre wie Michael Woodford umtun. ${ }^{5}$ Verhaltensökonomische Ansätze als grundlegendes Unterscheidungsmerkmal zur ,herrschenden Lehre" zu reklamieren, erscheint angesichts der Entwicklungen im Fach der letzten 15 Jahre nicht mehr angemessen zu sein. Wenn man aber anerkennt, dass die „Betrachtung von konventionellen Handlungsroutinen, eingeschränkter Rationalität und alternativen Entscheidungsverfahren " längst Teil des etablierten Forschungsprogramm ist, scheint sich das definierende Charakteristikum auf die oben diskutierte Rolle der Erwartungen zu reduzieren.

Konkreter wird der Text, wenn es um die wirtschaftspolitischen Implikationen geht: „Von besonderer Bedeutung für die Wirtschaftspolitik in Rahmen der Theorie der Marktteilnahme sind dabei Institutionen, die die Strategie- und Handlungsunsicherheit der Marktakteure reduzieren und damit ihre Handlungen vorhersehbarer machen: dies können Systeme fester Wechselkurse und Kollektivvertragssysteme sein, die die Bewertungsvolatilitäten reduzieren, vor allem aber sind kooperationsfördernde Institutionen notwendig, um die in den Handlungsinterdependenzen angelegten Handlungsblockaden - so kann beispielsweise eine auf expansive Intervention angelegte Finanzpolitik durch eine restriktiv ausgerichtete Geldpolitik blockiert werden, wenn die Notenbank inflationäre Begleiterscheinungen der Finanzpolitik erwartetzu lösen." (Heise 2019) Auch hier schließt sich eine Reihe von Fragen an. Dass ein System fester Wechselkurse die Unsicherheit reduziert, ist nach den katastrophalen Erfahrungen der Südländer in der Eurokrise eine eigentümliche Behauptung. Und die Forderung nach einer Aufgabe der Unabhängigkeit der Zentralbanken müsste, auch wenn sie so implizit formuliert wird, mit einer Erklärung einhergehen, wie der so oft beobachtete staatliche Missbrauch der Geldpolitik eingedämmt werden

\footnotetext{
5 Woodford hat eine ganze Reihe von jüngeren Forschungsarbeiten, die der Behavioral Macro zuzuordnen sind, bspw. ein Working Paper mit dem Titel ,Monetary Policy Analysis When Planning Horizons Are Finite“ (Woodford 2018), ein weiteres mit dem Titel „Rational Inattention and Sequential Information Sampling“ (Hébert und Woodford 2017). Damit ist Woodford bei weitem keine Ausnahme.
} 
soll. Zumindest erscheint es mir fraglich, ob die Staaten, die die Unabhängigkeit der Zentralbank wahren, unter höherer Unsicherheit im postkeynesianischen Sinne leiden als solche, die das nicht tun oder getan haben. Eine grundsätzlichere Frage ist dabei natürlich, inwiefern wirtschaftspolitische Implikationen aus einem Modell kommen können, das noch nach einer Fundierung sucht.

In seinem Fazit beklagt Heise die Marginalisierung postkeynesianischer Forschung. Eine postkeynesianische Erwartungstheorie, so Heise, sei ,,von unschätzbarem wissenschaftlichen (und wahrscheinlich auch gesellschaftlichem) Nutzen“ (Heise 2019). Doch genau dieser wird aus den vorangehenden Erklärungen nicht deutlich. Mir als der Makro Außenstehendem ist unmittelbar plausibel, dass ein Wettstreit zwischen mehreren theoretischen Ansätzen besser ist als die Hegemonie eines einzigen Ansatzes. Doch auch nach gründlicher Lektüre des Textes von Heise ist mir nicht klar, ob der Postkeynesianismus ein Kandidat für diesen Wettstreit ist. Heise ist sicher, dass der Postkeynesianismus nicht marginalisiert würde, ,wenn der ,Markt für wirtschaftswissenschaftliche Ideen` nur einigermaßen vernünftig funktionieren würde“ (Heise 2019). Mir fehlen überzeugende Argumente dafür, dass es nicht sogar Evidenz für die Funktionstüchtigkeit des Marktes für Ideen sein könnte, dass der Postkeynesianismus so selten vertreten wird. Projekte wie das von Farmer verfolgte Unterfangen, postkeynesianische Ideen in die Makroökonomik zu reintegrieren erscheinen mir vorbehaltlos unterstützenswert, doch ist keineswegs gesagt, ob dieses Projekt von Erfolg gekrönt sein wird. Doch ist dies genau der Wettbewerb, dem sich die postkeynesianischen Ideen stellen müssen. Sie einfach wie eine vom Aussterben bedrohte Tierart für bedingungslos schützens- und bewahrenswert zu erklären, wie Heises Text an manchen Stellen suggeriert, überzeugt mich nicht.

„Who is a Post-Keynesian? Who knows? Who cares?“, fragt Roger Farmer in seinem Blog. ${ }^{6}$ Dieser Haltung stimme ich zu. Es kommt nicht darauf an, irgendein Label zu bewahren oder zu schützen, sondern das Interessante und Hilfreiche in der postkeynesianischen Forschung für den modernen Forschungsdiskurs zugänglich zu machen. ${ }^{7}$ Wenn das bedeutet, dass die postkeynesianischen Ideen vom Mainstream geschluckt werden, ist das kein Verlust, sondern ein Fortschritt.

\section{Literatur}

Becker, J. (2017a). Die Plurale Ökonomik setzt ihren Erfolg aufs Spiel, Makronom. https://makronom.de/ volkswirtschaftslehre-die-plurale-oekonomik-setzt-ihren-erfolg-aufs-spiel-22399. Zugegriffen: 15. Februar 2019

Becker, J. (2017b). Das ,richtige“ Maß an Pluralität und das Problem des fehlenden Adressaten. Wirtschaftsdienst, 12, 835-838.

Farmer, R.E. A. (2017). Post-Keynesian dynamic stochastic general equilibrium theory. European Journal of Economics and Economic Policies: Intervention, 14(2), 173-185.

\footnotetext{
6 http://www.rogerfarmer.com/rogerfarmerblog/2017/7/30/who-is-a-post-keynesian

7 Auch Mervyn King, der ehemalige Präsident der Bank of England und selbst bekennender Postkeynesianer, fordert keine Rückkehr zu den alten keynesianischen Modellen: „The neoclassical ,optimising ' model does a better job of explaining saving and investment decisions. It recognises that when a household can borrow and save, the constraint on its spending is its income over its lifetime, not its current income." (S. 309) Doch das neoklassische Modell mache den Fehler, radikale Unsicherheit zu vernachlässigen - ein weiteres Argument, das sich auf die Erwartungsbildung bezieht (King 2016).
} 
Hébert, B., \& Woodford, M. (2017). Rational Inattention and Sequential Information Sampling. NBER Working Paper No. 23787.

Heise, A. (2019). Postkeynesianismus. List Forum für Wirtschafts- und Finanzpolitik. https://doi.org/10. 1007/s41025-019-00114-x.

King, M. (2016). The End of Alchemy, Little, Brown

Krugman, P. (1994). The fall and rise of development economics. http://web.mit.edu/krugman/www/ dishpan.html. Zugegriffen: 15. Februar 2019

Lawrence, Christiano, J., Eichenbaum, M.S., \& Trabandt, M. (2018). On DSGE models. Journal of Economic Perspectives, 32(3), 113-140.

Reis, R. (2018). Is something really wrong with macroeconomics?, Oxford Review of Economic Policy, $34(1-2), 132-155$.

Woodford, M. (2018). Monetary policy analysis when planning horizons are finite. NBER Working Paper No. 24692. https://doi.org/10.3386/w24692 